\title{
Hepatic Steatosis Index and Chronic Kidney Disease among Middle-Aged Individuals: A Large-Scale Study in Japan
}

\author{
Hirotaka Ochiai $\mathbb{D}^{1},{ }^{1}$ Takako Shirasawa, ${ }^{1}$ Takahiko Yoshimoto, ${ }^{1}$ Satsue Nagahama, ${ }^{2}$ \\ Ken Sakamoto, ${ }^{1}$ Minami Azuma, ${ }^{1}$ and Akatsuki Kokaze ${ }^{1}$ \\ ${ }^{1}$ Department of Hygiene, Public Health and Preventive Medicine, Showa University School of Medicine, Tokyo, Japan \\ ${ }^{2}$ All Japan Labor Welfare Foundation, Tokyo, Japan \\ Correspondence should be addressed to Hirotaka Ochiai; h-ochiai@med.showa-u.ac.jp
}

Received 30 March 2021; Revised 20 May 2021; Accepted 3 June 2021; Published 10 June 2021

Academic Editor: Zhengwen Liu

Copyright ( 2021 Hirotaka Ochiai et al. This is an open access article distributed under the Creative Commons Attribution License, which permits unrestricted use, distribution, and reproduction in any medium, provided the original work is properly cited.

\begin{abstract}
Background. Though nonalcoholic fatty liver disease (NAFLD) is related to chronic kidney disease (CKD), it is unclear whether the hepatic steatosis index (HSI), a screening tool for NAFLD, is related to CKD. The present study investigated the relationship between HSI and CKD among middle-aged individuals in Japan. Methods. Subjects were adults (aged 40-64 years) who received an annual health checkup in Japan between April 2013 and March 2014. Height and weight were measured, and venous blood samples were obtained to determine alanine aminotransferase (ALT), aspartate aminotransferase (AST), and creatinine levels. HSI was calculated by the following formula: HSI $=8 \times$ ALT/AST ratio + body mass index $(+2$, if diabetes; +2 , if female $)$. CKD was defined as an estimated glomerular filtration rate $<60 \mathrm{~mL} / \mathrm{min} / 1.73 \mathrm{~m}^{2}$ and/or urinary protein of $\geq(+)$. Logistic regression analysis was performed to estimate the odds ratio (OR) and its 95\% confidence interval (CI) for CKD. Results. Data of 94,893 adults were analyzed. Compared with men with an HSI < 30, men with $30 \leq$ HSI $\leq 36$ (OR: 1.50, 95\% CI: 1.40-1.61) and HSI $>36$ (OR: 2.14, 95\% CI: 1.99-2.31) had significantly higher ORs for CKD. Moreover, there was a significant doseresponse relationship between HSI and CKD $(P$ for trend $<0.001)$. Even after adjusting for confounders, the significant results persisted. These findings in men were similar to those in women. Conclusions. This study showed that the HSI was associated with CKD among middle-aged adults in Japan. Additionally, a dose-response relationship of HSI to CKD was observed. The present study suggested that it might be useful to monitor the HSI among middle-aged individuals to detect CKD at an early stage.
\end{abstract}

\section{Introduction}

Chronic kidney disease $(\mathrm{CKD})$ is reported to be a risk factor for cardiovascular disease (CVD) and CVD mortality $[1,2]$. Moreover, the progression of $\mathrm{CKD}$ is inevitable, resulting finally in end-stage renal disease (ESRD) [3]. ESRD substantially increases the risk of death [4]. With an estimated prevalence of CKD at $8-16 \%$ worldwide [5], CKD is a global public health problem.

A recent meta-analysis showed that patients with nonalcoholic fatty liver disease (NAFLD) have a higher risk of CKD than those without NAFLD, and the association between NAFLD and CKD is stronger in the Asian population than in the European population [6]. Therefore, those with NAFLD, especially in Asia, must be monitored for the early detection of CKD. Although histologic staging and grading using biopsy are the gold standard for the diagnosis of NAFLD [7], it is not feasible to perform liver biopsy solely to identify patients with NAFLD who are at high risk of developing CKD in the general population.

The hepatic steatosis index (HSI) was reported as a simple, efficient screening tool for NAFLD [8]. If the HSI is associated with CKD, it might be possible to use HSI for the identification of populations at high risk of CKD. Although we previously reported the association between the aspartate aminotransferase (AST) to alanine aminotransferase (ALT) ratio and CKD [9], it is unclear whether HSI including body mass index (BMI), diabetes, and sex in addition to AST and ALT is associated with CKD. Accordingly, 
this study investigated the relationship between HSI and CKD among middle-aged individuals in Japan.

\section{Subjects and Methods}

2.1. Subjects. Subjects in this study were adults aged 40-64 years who received an annual medical examination conducted by the "All Japan Labor Welfare Foundation," a health service provider in Japan, between April 2013 and March 2014. Of the 310,577 subjects, 310,498 consented to the use of their medical examination data for the present study. Of these participants, the measurement of serum creatinine (SCr) levels was performed for 129,910. Because 35,017 participants with missing data were excluded from the analysis, 94,893 participants were analyzed in this study. The present study was a cross-sectional survey that used the data including the study subjects, and several studies that used the data have been reported [9-15].

Written informed consent for the use of the medical examination data in our study was obtained from each participant. This study protocol was approved by the Medical Ethics Committee of the Showa University School of Medicine (Approval No. 2133) and the Ethics Committee of the All Japan Labor Welfare Foundation (Approval No. 2-1-0003).

2.2. Data Collection. Information on the smoking status, alcohol intake, and physical activity was collected from a self-administered questionnaire that is recommended for specific health examinations by the Ministry of Health, Labour, and Welfare.

Height and weight were measured in increments of $0.1 \mathrm{~cm}$ and $0.1 \mathrm{~kg}$, respectively. BMI was calculated as weight $(\mathrm{kg}) /$ height $^{2}\left(\mathrm{~m}^{2}\right)$. The measurement of blood pressure (systolic blood pressure (SBP) and diastolic blood pressure (DBP)) was conducted in the sitting position by an automated machine (HEM-907, Omron, Kyoto, Japan).

Blood samples were drawn and transported to an external laboratory (SRL, Tokyo, Japan). Blood glucose, hemoglobin A1c (HbA1c), high-density lipoprotein cholesterol (HDLC), low-density lipoprotein cholesterol (LDL-C), triglyceride (TG), ALT, AST, and SCr levels were measured within 24 hours of blood collection. The detail of the measurements was described in our previous studies [9-15]. The estimated glomerular filtration rate (eGFR) was calculated with the following formula: $\mathrm{eGFR}=194 \times \mathrm{SCr}(\mathrm{mg} / \mathrm{dL})^{-1.094} \times$ age $(\text { years })^{-0.287}$ (if female $\times 0.739$ ) [16]. Urine analysis was performed using the dipstick test, and the test results were reported as $(-),( \pm),(+),(++)$, or $(+++)$.

2.3. Definitions. Hypertension was determined as $\mathrm{SBP} \geq$ $140 \mathrm{mmHg}, \mathrm{DBP} \geq 90 \mathrm{mmHg}$, or the use of antihypertensive medication [17]. Dyslipidemia was regarded as LDL $-\mathrm{C} \geq$ $140 \mathrm{mg} / \mathrm{dL}, \mathrm{HDL}-\mathrm{C}<40 \mathrm{mg} / \mathrm{dL}, \mathrm{TG} \geq 150 \mathrm{mg} / \mathrm{dL}$, or the use of an antidyslipidemic drug [18]. Diabetes was defined as a fasting plasma glucose ( $\geq 8$ hours after the last caloric intake [19]) $\geq 126 \mathrm{mg} / \mathrm{dL}$, a random plasma glucose $\geq 200$ $\mathrm{mg} / \mathrm{dL}, \mathrm{HbA1c}$ (National Glycohemoglobin Standardization Program) $\geq 6.5 \%$, or the use of an antidiabetic drug [20].
The HSI was calculated by the following formula: HSI = $8 \times \mathrm{ALT} / \mathrm{AST}$ ratio $+\mathrm{BMI}(+2$, if diabetes; +2 , if female) [8]. HSI was divided into three categories $(<30,30-36$, and $>36$ ) because $\mathrm{HSI}<30$ rules out NAFLD and HSI $>36$ rules in NAFLD $[8,21]$. In accordance with recent studies [22-24], $\mathrm{CKD}$ was determined as $\mathrm{eGFR}<60 \mathrm{~mL} / \mathrm{min} / 1.73 \mathrm{~m}^{2}$ and/or urinary protein of $\geq(+)$.

2.4. Statistical Analyses. Data are shown as mean (standard deviation) or $n$ (\%). The data of SCr, ALT, and AST are presented as medians $(25,75$ th percentile) because the distributions were highly skewed. Wilcoxon's rank-sum test, unpaired $t$-test, or chi-square test were used for the comparison of characteristics between two groups (the CKD group and the no-CKD group). Logistic regression analysis was employed to estimate the odds ratio (OR) and its 95\% confidence interval (CI) for CKD. First, we calculated a crude OR. Second, age, smoking status, alcohol intake, physical activity, hypertension, and dyslipidemia were adjusted for as confounding factors [25-27]. Because diabetes was included in HSI, it was not included as a confounder in the model for examining the relationship between HSI and CKD.

All statistical analyses were conducted using Statistical Analysis System (SAS) software (version 9.4; SAS Institute Inc., Cary, NC, USA), and a $P$ value less than 0.05 was regarded as statistically significant.

\section{Results}

The mean age of this study's participants $(n=94,893)$ was 50.6 years. The proportion of men $(n=65,850)$ was $69.4 \%$, while that of women $(n=29,043)$ was $30.6 \%$. The prevalence of CKD overall, in men, and in women was $8.3 \%, 8.0 \%$, and $9.2 \%$, respectively.

The characteristics of the study participants are shown by sex in Tables 1 and 2. Age and BMI were significantly higher in the CKD group than in the no-CKD group. There were significant differences between the CKD and no-CKD groups in smoking status, alcoholic intake, and physical activity. The proportions of those with hypertension, dyslipidemia, and diabetes were significantly higher in the CKD group than in the no-CKD group. HSI in the CKD group was significantly higher than that in the no-CKD group.

Crude and adjusted ORs for CKD were calculated and reported by sex (Table 3). Compared with men with an HSI $<30$, men with $30 \leq \mathrm{HSI} \leq 36$ (OR: 1.50 , 95\% CI: 1.40 1.61) and HSI > 36 (OR: 2.14, 95\% CI: 1.99-2.31) had significantly higher ORs for CKD. These findings in men were similar to those in women (OR: $1.73,95 \%$ CI: 1.58-1.89; OR: $2.62,95 \%$ CI: $2.36-2.92$, respectively). In addition, there was a significant dose-response relationship between HSI and CKD in both men and women ( $P$ for trend $<0.001)$. Even after adjusting for confounders, these significant results persisted.

Next, participants were divided into four groups according to quartiles of HSI levels, and the association between HSI and CKD was evaluated for the sensitivity analysis (Table 4). A higher level of HSI was associated with a significantly higher OR for CKD among men and women $(P$ for 
TABLE 1: Characteristics of study participants (men).

\begin{tabular}{|c|c|c|c|}
\hline & No CKD $(n=60,608)$ & $\mathrm{CKD}(n=5,242)$ & $P$ value $^{\mathrm{a}}$ \\
\hline Age (years) & $50.3(7.0)$ & $54.1(7.0)$ & $<0.001$ \\
\hline BMI $\left(\mathrm{kg} / \mathrm{m}^{2}\right)$ & $23.6(3.5)$ & $25.1(3.9)$ & $<0.001$ \\
\hline \multicolumn{4}{|l|}{ Smoking status ( $n(\%))$} \\
\hline None & $21,771(35.9)$ & $2,298(43.8)$ & \multirow[t]{3}{*}{$<0.001$} \\
\hline Former & $11,817(19.5)$ & $1,237(23.6)$ & \\
\hline Current & $27,020(44.6)$ & $1,707(32.6)$ & \\
\hline \multicolumn{4}{|l|}{ Alcohol intake $(n(\%))$} \\
\hline None & $16,058(26.5)$ & $1,712(32.7)$ & \multirow[t]{3}{*}{$<0.001$} \\
\hline Sometimes & $18,532(30.6)$ & $1,717(32.8)$ & \\
\hline Everyday & $26,018(42.9)$ & $1,813(34.6)$ & \\
\hline \multicolumn{4}{|l|}{ Physical activity $(n(\%))$} \\
\hline$<60 \mathrm{~min} /$ day & $40,365(66.6)$ & $3,634(69.3)$ & \multirow[t]{2}{*}{$<0.001$} \\
\hline$\geq 60 \mathrm{~min} /$ day & $20,243(33.4)$ & $1,608(30.7)$ & \\
\hline Hypertension (n (\%)) & $22,034(36.4)$ & $2,524(48.2)$ & $<0.001$ \\
\hline Dyslipidemia $(n(\%))$ & $31,969(52.8)$ & $3,182(60.7)$ & $<0.001$ \\
\hline Diabetes $(n(\%))$ & $4,878(8.1)$ & $963(18.4)$ & $<0.001$ \\
\hline Creatinine (mg/dL) & $0.80(0.73,0.90)$ & $1.07(1.00,1.13)$ & $<0.001$ \\
\hline eGFR $\left(\mathrm{mL} / \mathrm{min} / 1.73 \mathrm{~m}^{2}\right)$ & $81.4(13.2)$ & $60.6(15.8)$ & $<0.001$ \\
\hline $\operatorname{ALT}(\mathrm{U} / \mathrm{L})$ & $22(17,32)$ & $24(17,34)$ & $<0.001$ \\
\hline AST (U/L) & $22(19,27)$ & $23(19,29)$ & $<0.001$ \\
\hline HSI & $32.4(5.5)$ & $34.2(5.9)$ & $<0.001$ \\
\hline
\end{tabular}

ALT: alanine aminotransferase; AST: aspartate aminotransferase; BMI: body mass index; CKD: chronic kidney disease; eGFR: estimated glomerular filtration rate; HSI: hepatic steatosis index. Values are mean (standard deviation) or median (25th percentile, 75 th percentile) except where indicated, $n$ (\%). ${ }^{2}$ Wilcoxon's rank-sum test, unpaired $t$-test, or chi-squared test.

trend $<0.001)$. The results persisted even when the confounding factors were adjusted.

\section{Discussion}

We investigated the relationship of HSI to CKD in middleaged individuals in Japan. A higher level of HSI was associated with a significantly higher OR for CKD in both men and women. This is the first study to examine the association between HSI and CKD, to the best of our knowledge. However, our results need to be treated with caution.

In this study, the prevalence in men (aged 40-49 and 50-59 years) with eGFR $<60 \mathrm{~mL} / \mathrm{min} / 1.73 \mathrm{~m}^{2}$ was $2.8 \%$ and $6.6 \%$, while that in women was $4.5 \%$ and $10.8 \%$, respectively. A previous study conducted in Japan showed that the prevalence among those aged 40-49 and 50-59 years was $4.0 \%$ and $7.5 \%$ in men, while the prevalence was $2.6 \%$ and $6.8 \%$ in women, respectively [28]. A recent study reported that the prevalence was $4.3 \%$ among men aged $40-49$ years and $11.0 \%$ among those aged $50-59$ years, while the prevalence was $3.1 \%$ among women aged $40-49$ years and $10.0 \%$ among those aged 50-59 years [29]. The difference between our results and those of the other studies might be due to the difference in the data collection periods. Data in this study were collected from 2013 to 2014, while data in the other studies were during 2000-2004 and in 2017. A previous survey performed during 2001-2002 reported that the prevalence of CKD was $13.2 \%$ among middle-aged people (mean age: 52.1 years) in Korea [30]. A recent cross-sectional study based on data collected from 2015 to 2016 showed that the prevalence of CKD was $7.3 \%$ in the middle-aged Chinese population (aged 40-59 years) [31]. The previous study's prevalence is different from that $(8.3 \%)$ in our study among individuals aged 40-64 years (mean age, 50.6 years), as the current study is based on the data obtained from April 2013 to March 2014. The reasons for this difference in the prevalence could be owing to differences in age, country, and study period.

In our study, HSI $>36$ had a significantly higher OR for CKD than HSI $<30$. HSI is reported to be a screening tool for NAFLD [8], and HSI > 36 ruled in NAFLD while HSI < 30 ruled out NAFLD. NAFLD has been shown to be associated with CKD [6]. Furthermore, a recent study reported that CKD is likely to be more common in adults with NAFLD than in those without it [32]. Patients with NAFLD have insulin resistance and hypercoagulability (high fibrinogen, factor VII, and von Willebrand factor levels), which are risk factors for CKD [33]. These studies corroborate our study findings to be reasonable, though the risk factors (insulin resistance and hypercoagulability) were not considered in the present study.

The present study results suggested that it is possible to use HSI for the identification of individuals at high risk of CKD. For instance, it could be effective to use HSI in addition to metabolic syndrome (MS), which was reported to be associated with CKD $[34,35]$, to identify the population at high 
TABLE 2: Characteristics of study participants (women).

\begin{tabular}{|c|c|c|c|}
\hline & No CKD $(n=26,375)$ & $\mathrm{CKD}(n=2,668)$ & $P$ value $^{\mathrm{a}}$ \\
\hline Age (years) & $50.4(6.9)$ & $53.4(6.6)$ & $<0.001$ \\
\hline BMI $\left(\mathrm{kg} / \mathrm{m}^{2}\right)$ & $22.1(3.7)$ & $23.4(4.0)$ & $<0.001$ \\
\hline \multicolumn{4}{|l|}{ Smoking status $(n(\%))$} \\
\hline None & $19,805(75.1)$ & $1,755(65.8)$ & \multirow[t]{3}{*}{$<0.001$} \\
\hline Former & $1,770(6.7)$ & $324(12.1)$ & \\
\hline Current & $4,800(18.2)$ & $589(22.1)$ & \\
\hline \multicolumn{4}{|l|}{ Alcohol intake $(n(\%))$} \\
\hline None & $14,318(54.3)$ & $1,292(48.4)$ & \multirow[t]{3}{*}{$<0.001$} \\
\hline Sometimes & $7,905(30.0)$ & $776(29.1)$ & \\
\hline Everyday & $4,152(15.7)$ & $600(22.5)$ & \\
\hline \multicolumn{4}{|l|}{ Physical activity $(n(\%))$} \\
\hline$<60 \mathrm{~min} /$ day & $18,511(70.2)$ & $1,809(67.8)$ & \multirow[t]{2}{*}{$<0.001$} \\
\hline$\geq 60 \mathrm{~min} /$ day & $7,864(29.8)$ & $859(32.2)$ & \\
\hline Hypertension (n (\%)) & $7,625(28.9)$ & $982(36.8)$ & $<0.001$ \\
\hline Dyslipidemia ( $n(\%))$ & $10,775(40.9)$ & $1,353(50.7)$ & $<0.001$ \\
\hline Diabetes $(n(\%))$ & $881(3.3)$ & $191(7.2)$ & $<0.001$ \\
\hline Creatinine (mg/dL) & $0.60(0.56,0.69)$ & $0.82(0.80,0.90)$ & $<0.001$ \\
\hline eGFR $\left(\mathrm{mL} / \mathrm{min} / 1.73 \mathrm{~m}^{2}\right)$ & $81.4(13.0)$ & $56.5(11.4)$ & $<0.001$ \\
\hline $\operatorname{ALT}(\mathrm{U} / \mathrm{L})$ & $15(12,20)$ & $18(14,25)$ & $<0.001$ \\
\hline AST (U/L) & $20(17,23)$ & $22(18,26)$ & $<0.001$ \\
\hline HSI & $30.9(5.0)$ & $32.9(5.7)$ & $<0.001$ \\
\hline
\end{tabular}

ALT: alanine aminotransferase; AST: aspartate aminotransferase; BMI: body mass index; CKD: chronic kidney disease; eGFR: estimated glomerular filtration rate; HSI: hepatic steatosis index. Values are mean (standard deviation) or median (25th percentile, 75 th percentile) except where indicated, $n$ (\%). ${ }^{2}$ Wilcoxon's rank-sum test, unpaired $t$-test, or chi-squared test.

TABle 3: Association between hepatic steatosis index and chronic kidney disease by sex.

\begin{tabular}{|c|c|c|c|c|}
\hline & Total $(N)$ & CKD $(n(\%))$ & $\begin{array}{c}\text { Crude } \\
\text { OR }(95 \% \mathrm{CI})\end{array}$ & $\begin{array}{c}\text { Adjusted } \\
\text { OR }(95 \% \mathrm{CI})\end{array}$ \\
\hline \multicolumn{5}{|l|}{ Men $(n=65,850)$} \\
\hline $36<$ HSI & 15,606 & $1,756(11.3)$ & $2.14(1.99-2.31)$ & $2.13(1.97-2.31)$ \\
\hline $30 \leq \mathrm{HSI} \leq 36$ & 26,566 & $2,164(8.2)$ & $1.50(1.40-1.61)$ & $1.40(1.30-1.51)$ \\
\hline \multirow[t]{2}{*}{$\mathrm{HSI}<30$} & 23,678 & $1,322(5.6)$ & 1.00 & 1.00 \\
\hline & & & $P$ for trend $<0.001$ & $P$ for trend $<0.001$ \\
\hline \multicolumn{5}{|c|}{ Women $(n=29,043)$} \\
\hline $36<$ HSI & 4,357 & $661(15.2)$ & $2.62(2.36-2.92)$ & $2.42(2.17-2.71)$ \\
\hline $30 \leq \mathrm{HSI} \leq 36$ & 10,387 & $1,094(10.5)$ & $1.73(1.58-1.89)$ & $1.56(1.42-1.72)$ \\
\hline \multirow[t]{2}{*}{$\mathrm{HSI}<30$} & 14,299 & $913(6.4)$ & 1.00 & 1.00 \\
\hline & & & $P$ for trend $<0.001$ & $P$ for trend $<0.001$ \\
\hline
\end{tabular}

CI: confidence interval; CKD: chronic kidney disease; HSI: hepatic steatosis index; OR: odds ratio. Adjusted for age, smoking status, alcohol intake, physical activity, hypertension, and dyslipidemia.

risk of CKD. Of 76,920 participants with data on waist circumference and fasting plasma glucose in this study, the proportion of individuals with HSI $>36$ and MS defined by the criteria of the committee to evaluate diagnostic standards for metabolic syndrome [36-38] was $8.3 \%$. Furthermore, the proportion was $11.5 \%$ among those with HSI $>36$ and without MS, while the proportion was $5.1 \%$ among those with HSI $\leq 36$ and MS. Thus, it might be feasible to target populations $(8.3 \%)$ with both HSI $>36$ and MS compared with those $(19.8 \%)$ with HSI $>36$.

A dose-response relationship between HSI and CKD was observed in the present study. HSI has been shown to be associated with C-reactive protein (CRP) levels [21, 39]. CRP is a very sensitive marker of inflammation [40], which contributes to the initiation and progression of CKD [41]. Previous studies have also shown that CRP is associated with 
TABLE 4: Odds ratios and their 95\% confidence intervals for chronic kidney disease in the group with different levels of hepatic steatosis index by sex.

\begin{tabular}{|c|c|c|c|c|}
\hline & Total $(N)$ & $\mathrm{CKD}(n(\%))$ & $\begin{array}{c}\text { Crude } \\
\text { OR }(95 \% \mathrm{CI})\end{array}$ & $\begin{array}{c}\text { Adjusted } \\
\text { OR }(95 \% \mathrm{CI})\end{array}$ \\
\hline \multicolumn{5}{|l|}{ Men $(n=65,850)$} \\
\hline Q4 $(35.8<$ HSI $)$ & 16,196 & $1,804(11.1)$ & $2.33(2.14-2.54)$ & $2.28(2.08-2.49)$ \\
\hline Q3 $(31.8<\mathrm{HSI} \leq 35.8)$ & 16,243 & $1,377(8.5)$ & $1.72(1.58-1.88)$ & $1.57(1.43-1.72)$ \\
\hline Q2 $(28.5<\mathrm{HSI} \leq 31.8)$ & 16,906 & $1,219(7.2)$ & $1.45(1.32-1.58)$ & $1.36(1.24-1.49)$ \\
\hline \multirow[t]{2}{*}{ Q1 (HSI $\leq 28.5)$} & 16,505 & $842(5.1)$ & 1.00 & 1.00 \\
\hline & & & $P$ for trend $<0.001$ & $P$ for trend $<0.001$ \\
\hline \multicolumn{5}{|l|}{ Women $(n=29,043)$} \\
\hline Q4 $(33.5<$ HSI $)$ & 7,326 & $1,026(14.0)$ & $2.62(2.33-2.95)$ & $2.35(2.08-2.65)$ \\
\hline Q3 $(30.1<\mathrm{HSI} \leq 33.5)$ & 7,101 & $706(9.9)$ & $1.78(1.57-2.01)$ & $1.61(1.42-1.83)$ \\
\hline Q2 $(27.6<\mathrm{HSI} \leq 30.1)$ & 7,289 & $507(7.0)$ & $1.20(1.05-1.37)$ & $1.18(1.03-1.35)$ \\
\hline \multirow[t]{2}{*}{ Q1 $(\mathrm{HSI} \leq 27.6)$} & 7,327 & $429(5.9)$ & 1.00 & 1.00 \\
\hline & & & $P$ for trend $<0.001$ & $P$ for trend $<0.001$ \\
\hline
\end{tabular}

CI: confidence interval; CKD: chronic kidney disease; HSI: hepatic steatosis index; OR: odds ratio; Q1: quartile 1; Q2: quartile 2; Q3: quartile 3; Q4: quartile 4. Adjusted for age, smoking status, alcohol intake, physical activity, hypertension, and dyslipidemia.

diminished renal filtration [40] and CKD [42]. Because there were no data on CRP in this study, further studies will be needed to elucidate the influence of CRP on the relationship between HSI and CKD.

This study included the large-scale sample (more than 90,000 participants) in Japan, which was the strength of our study. However, a few limitations are present. First, some potential confounding factors that were not controlled for might affect our study findings. For instance, family history of CKD and low birth weight are known risk factors for CKD [43]. Because the information on these factors was unavailable in this study, it is necessary to control for them in future studies. Second, this study included data on middle-aged adults who received an annual health checkup in Japan. Thus, it would be difficult to generalize our study findings to other populations. Finally, this study had a cross-sectional survey design. Therefore, the causal relationship between HSI and CKD could not be established. Cohort studies will be needed to examine the temporal relationship between HSI and CKD.

\section{Conclusion}

The present study showed that the HSI was associated with CKD among middle-aged individuals in Japan. Moreover, the dose-response relationship of HSI with CKD was found. This study's findings suggest that HSI might be useful to detect CKD at an early stage, by the monitoring of HSI among middle-aged populations.

\section{Data Availability}

Data are available on reasonable request and only after approval by the Ethics Committee of the All Japan Labor Welfare Foundation.

\section{Disclosure}

Showa University and All Japan Labor Welfare Foundation had no role in the present study design, analysis, and interpretation of data.

\section{Conflicts of Interest}

The authors declare that there is no conflict of interest regarding the publication of this article.

\section{Acknowledgments}

The authors thank Dr. Nobuo Yanagisawa, Dr. Takeshi Kawaguchi, and Mr. Yutaka Hoshina in the All Japan Labor Welfare Foundation for their support. We would like to thank Editage (https://www.editage.com) for English language editing. This work was supported by Showa University and All Japan Labor Welfare Foundation.

\section{References}

[1] C. Meisinger, A. Doring, H. Lowel, and K. S. Group, "Chronic kidney disease and risk of incident myocardial infarction and all-cause and cardiovascular disease mortality in middle-aged men and women from the general population," European Heart Journal, vol. 27, no. 10, pp. 1245-1250, 2006.

[2] N. I. Parikh, S. J. Hwang, M. G. Larson, D. Levy, and C. S. Fox, "Chronic kidney disease as a predictor of cardiovascular disease (from the Framingham Heart Study)," The American Journal of Cardiology, vol. 102, no. 1, pp. 47-53, 2008.

[3] U. A. Sharaf El Din, M. M. Salem, and D. O. Abdulazim, "Stop chronic kidney disease progression: time is approaching," World J Nephrol, vol. 5, no. 3, pp. 258-273, 2016.

[4] A. S. Go, G. M. Chertow, D. Fan, C. E. McCulloch, and C. Y. $\mathrm{Hsu}$, "Chronic kidney disease and the risks of death, cardiovascular events, and hospitalization," The New England Journal of Medicine, vol. 351, no. 13, pp. 1296-1305, 2004. 
[5] V. Jha, G. Garcia-Garcia, K. Iseki et al., "Chronic kidney disease: global dimension and perspectives," Lancet, vol. 382, no. 9888, pp. 260-272, 2013.

[6] A. Mantovani, G. Zaza, C. D. Byrne et al., "Nonalcoholic fatty liver disease increases risk of incident chronic kidney disease: a systematic review and meta-analysis," Metabolism, vol. 79, pp. $64-76,2018$.

[7] J. Lee, J. Ha, K. Jo et al., "Male-specific association between subclinical hypothyroidism and the risk of non-alcoholic fatty liver disease estimated by hepatic steatosis index: Korea National Health and Nutrition Examination Survey 2013 to 2015," Scientific Reports, vol. 8, no. 1, p. 15145, 2018.

[8] J. H. Lee, D. Kim, H. J. Kim et al., "Hepatic steatosis index: a simple screening tool reflecting nonalcoholic fatty liver disease," Digestive and Liver Disease, vol. 42, no. 7, pp. 503-508, 2010.

[9] H. Ochiai, T. Shirasawa, T. Yoshimoto et al., "Elevated alanine aminotransferase and low aspartate aminotransferase/alanine aminotransferase ratio are associated with chronic kidney disease among middle-aged women: a cross-sectional study," BMC Nephrology, vol. 21, no. 1, p. 471, 2020.

[10] H. Ochiai, T. Shirasawa, T. Yoshimoto et al., "Association of the combination of weight gain after 20 years of age and current obesity with chronic kidney disease in Japan: a crosssectional study," BMJ Open, vol. 9, no. 6, article e027752, 2019.

[11] T. Shirasawa, H. Ochiai, T. Yoshimoto et al., "Associations between normal weight central obesity and cardiovascular disease risk factors in Japanese middle-aged adults: a crosssectional study," Journal of Health, Population, and Nutrition, vol. 38, no. 1, p. 46, 2019.

[12] T. Shirasawa, H. Ochiai, T. Yoshimoto et al., "Cross-sectional study of associations between normal body weight with central obesity and hyperuricemia in Japan," BMC Endocrine Disorders, vol. 20, no. 1, p. 2, 2020.

[13] T. Yoshimoto, H. Ochiai, T. Shirasawa et al., "Association between serum lipids and low back pain among a middleaged Japanese population: a large-scale cross-sectional study," Lipids in Health and Disease, vol. 17, no. 1, p. 266, 2018.

[14] T. Yoshimoto, H. Ochiai, T. Shirasawa et al., "Clustering of lifestyle factors and its association with low back pain: a cross-sectional study of over 400,000 Japanese adults," Journal of Pain Research, vol. Volume 13, pp. 1411-1419, 2020.

[15] T. Yoshimoto, H. Ochiai, T. Shirasawa et al., "Sex differences in the association of metabolic syndrome with low back pain among middle-aged Japanese adults: a large-scale crosssectional study," Biology of Sex Differences, vol. 10, no. 1, p. 33, 2019.

[16] S. Matsuo, E. Imai, M. Horio et al., "Revised equations for estimated GFR from serum creatinine in Japan," American Journal of Kidney Diseases, vol. 53, no. 6, pp. 982-992, 2009.

[17] T. Otsuka, H. Takada, Y. Nishiyama et al., "Dyslipidemia and the risk of developing hypertension in a working-age male population," Journal of the American Heart Association, vol. 5, article e003053, 2016.

[18] K. Hara, M. Okada, D. Takagi et al., "Association between hypertension, dyslipidemia, and diabetes and prevalence of hearing impairment in Japan," Hypertension Research, vol. 43, no. 9, pp. 963-968, 2020.

[19] Y. Kabeya, M. Kato, A. Isogawa et al., "Descriptive epidemiology of diabetes prevalence and $\mathrm{HbAlc}$ distributions based on a self-reported questionnaire and a health checkup in the JPHC diabetes study," Journal of Epidemiology, vol. 24, no. 6, pp. 460-468, 2014.

[20] M. Yasuoka, I. Muraki, H. Imano et al., "Joint impact of muscle mass and waist circumference on type 2 diabetes in Japanese middle-aged adults: the Circulatory Risk in Communities Study (CIRCS)," Journal of Diabetes, vol. 12, no. 9, pp. 677$685,2020$.

[21] S. K. Kunutsor, S. J. L. Bakker, H. Blokzijl, and R. P. F. Dullaart, "Associations of the fatty liver and hepatic steatosis indices with risk of cardiovascular disease: interrelationship with age," Clinica Chimica Acta, vol. 466, pp. 54-60, 2017.

[22] Y. He, F. Li, F. Wang, X. Ma, X. Zhao, and Q. Zeng, "The association of chronic kidney disease and waist circumference and waist-to-height ratio in Chinese urban adults," Medicine (Baltimore), vol. 95, no. 25, article e3769, 2016.

[23] N. Nakagawa, T. Sofue, E. Kanda et al., "J-CKD-DB: a nationwide multicentre electronic health record-based chronic kidney disease database in Japan," Scientific Reports, vol. 10, no. 1, p. 7351, 2020.

[24] K. Nakamura, H. Nakagawa, Y. Murakami et al., "Smoking increases the risk of all-cause and cardiovascular mortality in patients with chronic kidney disease," Kidney International, vol. 88, no. 5, pp. 1144-1152, 2015.

[25] C. Guo, T. Tam, Y. Bo, L. Y. Chang, X. Q. Lao, and G. N. Thomas, "Habitual physical activity, renal function and chronic kidney disease: a cohort study of nearly 200000 adults," British Journal of Sports Medicine, vol. 54, no. 20, pp. 1225-1230, 2020.

[26] Y. Ohno, E. Ishimura, T. Naganuma et al., "Prevalence of and factors associated with chronic kidney disease (CKD) in Japanese subjects without notable chronic diseases, undergoing an annual health checkup," Kidney \& Blood Pressure Research, vol. 36, no. 1, pp. 139-148, 2012.

[27] K. Yamagata, K. Ishida, T. Sairenchi et al., "Risk factors for chronic kidney disease in a community-based population: a 10-year follow-up study," Kidney International, vol. 71, no. 2, pp. 159-166, 2007.

[28] E. Imai, M. Horio, T. Watanabe et al., "Prevalence of chronic kidney disease in the Japanese general population," Clinical and Experimental Nephrology, vol. 13, no. 6, pp. 621-630, 2009.

[29] M. Takeuchi, K. Shinkawa, M. Yanagita, and K. Kawakami, "Prevalence, recognition and management of chronic kidney disease in Japan: population-based estimate using a healthcare database with routine health checkup data," Clinical Kidney Journal, pp. 1-6, 2021.

[30] M. J. Kim, N. K. Lim, and H. Y. Park, "Relationship between prehypertension and chronic kidney disease in middle-aged people in Korea: the Korean genome and epidemiology study," BMC Public Health, vol. 12, no. 1, p. 960, 2012.

[31] S. S. Xu, J. Hua, Y. Q. Huang, and L. Shu, “Association between dietary patterns and chronic kidney disease in a middle-aged Chinese population," Public Health Nutrition, vol. 23, no. 6, pp. 1058-1066, 2020.

[32] J. Zeng, C. Sun, W. L. Sun et al., “Association between noninvasively diagnosed hepatic steatosis and chronic kidney disease in Chinese adults on their health check-up," Journal of Digestive Diseases, vol. 18, no. 4, pp. 229-236, 2017.

[33] G. Targher, M. B. Chonchol, and C. D. Byrne, "CKD and nonalcoholic fatty liver disease," American Journal of Kidney Diseases, vol. 64, no. 4, pp. 638-652, 2014. 
[34] J. H. Huh, D. Yadav, J. S. Kim et al., “An association of metabolic syndrome and chronic kidney disease from a 10-year prospective cohort study," Metabolism, vol. 67, pp. 54-61, 2017.

[35] Y. U. Kang, H. Y. Kim, J. S. Choi et al., "Metabolic syndrome and chronic kidney disease in an adult Korean population: results from the Korean National Health Screening," PLoS One, vol. 9, no. 5, article e93795, 2014.

[36] A. Morita, N. Aiba, M. Miyachi, S. Watanabe, and Saku Cohort Study, G, "The associations of eating behavior and dietary intake with metabolic syndrome in Japanese: Saku cohort baseline study," Journal of Physiological Anthropology, vol. 39, no. 1, p. 40, 2020.

[37] H. Ohnishi, S. Saitoh, H. Akasaka et al., "Incidence of hypertension in individuals with abdominal obesity in a rural Japanese population: the Tanno and Sobetsu study," Hypertension Research, vol. 31, no. 7, pp. 1385-1390, 2008.

[38] A. Tsubakimoto, I. Saito, T. Mannami et al., "Impact of metabolic syndrome on brachial-ankle pulse wave velocity in Japanese," Hypertension Research, vol. 29, no. 1, pp. 29-37, 2006.

[39] L. Sviklane, E. Olmane, Z. Dzerve, K. Kupcs, V. Pirags, and J. Sokolovska, "Fatty liver index and hepatic steatosis index for prediction of non-alcoholic fatty liver disease in type 1 diabetes," Journal of Gastroenterology and Hepatology, vol. 33, no. 1, pp. 270-276, 2018.

[40] E. M. Stuveling, H. L. Hillege, S. J. Bakker, R. O. Gans, P. E. De Jong, and D. De Zeeuw, "C-reactive protein is associated with renal function abnormalities in a non-diabetic population," Kidney International, vol. 63, no. 2, pp. 654-661, 2003.

[41] D. D. Waters and L. Vogt, "Lipids, inflammation, and chronic kidney disease: a SHARP perspective," Kidney International, vol. 93, no. 4, pp. 784-786, 2018.

[42] E. R. Fox, E. J. Benjamin, D. F. Sarpong et al., "The relation of C-reactive protein to chronic kidney disease in African Americans: the Jackson Heart Study," BMC Nephrology, vol. 11, no. 1, p. 1, 2010.

[43] Y. Kakitapalli, J. Ampolu, S. D. Madasu, and M. L. S. Sai Kumar, "Detailed review of chronic kidney disease," Kidney Dis (Basel), vol. 6, no. 2, pp. 85-91, 2020. 